\title{
HYPER-DUAL HORADAM QUATERNIONS
}

\author{
N. ROSA AIT-AMRANE, ISMAIL GOK, AND ELIF TAN
}

Received 28 February, 2021

\begin{abstract}
This paper deals with developing a new class of quaternions, called hyper-dual Horadam quaternions which are constructed from the quaternions whose components are hyper-dual Horadam numbers. We investigate the algebraic properties of these quaternions.
\end{abstract}

2010 Mathematics Subject Classification: 11B39; 11R52

Keywords: dual numbers, hyper-dual numbers, dual quaternions, Fibonacci numbers, hyper-dual Horadam quaternions

\section{INTRODUCTION}

Dual numbers were invented by Clifford [3] as an extension of the real numbers. The set of dual numbers is defined as

$$
\mathbb{D}=\left\{d=a+a^{*} \varepsilon \mid a, a^{*} \in \mathbb{R}\right\},
$$

where $\varepsilon$ is the dual unit with $\varepsilon \neq 0, \varepsilon^{2}=0$. Dual numbers have many interesting applications on mechanics, robotics, computer graphics, geometry, and physics. Especially, they are important to represent rigid body motions in 3D-space. The addition and multiplication of two dual numbers $d_{1}=a+a^{*} \varepsilon$ and $d_{2}=b+b^{*} \varepsilon$ are defined as

$$
d_{1}+d_{2}=(a+b)+\left(a^{*}+b^{*}\right) \varepsilon \quad \text { and } \quad d_{1} d_{2}=a b+\left(a b^{*}+a^{*} b\right) \varepsilon,
$$

respectively.

Similar to the quaternions, dual quaternions are defined by taking dual numbers instead of real numbers as coefficients. A dual quaternion $\widetilde{q}$ is defined as

$$
\widetilde{q}=d_{0}+d_{1} i+d_{2} j+d_{3} k,
$$

where $d_{0}, d_{1}, d_{2}, d_{3} \in \mathbb{D}$, and the elements $i, j, k$ satisfy the following noncommutative quaternion multiplication rules:

$$
i^{2}=j^{2}=k^{2}=i j k=-1 .
$$

Since any dual quaternion can be written as a dual number with real quaternion coefficients, it is constructed from eight real parameters. For the detailed information related to these numbers and their applications, we refer to $[3,10,16]$. 
Hyper-dual numbers can be seen as an extension of dual numbers in the same way that quaternions are an extension of complex numbers. To get an advantage on exact calculations of second (or higher) derivatives, Fike and Alanso [6,7] introduced the hyper-dual numbers. The set of hyper-dual numbers is defined by

$$
\mathbb{H I D}=\left\{D=a_{0}+a_{1} \varepsilon_{1}+a_{2} \varepsilon_{2}+a_{3} \varepsilon_{1} \varepsilon_{2} \mid a_{0}, a_{1}, a_{2}, a_{3} \in \mathbb{R}\right\},
$$

where the dual units $\varepsilon_{1}$ and $\varepsilon_{2}$ satisfy the following rules:

$$
\varepsilon_{1}^{2}=\varepsilon_{2}^{2}=\left(\varepsilon_{1} \varepsilon_{2}\right)^{2}=0, \quad \varepsilon_{1} \neq \varepsilon_{2}, \quad \varepsilon_{1} \neq 0, \varepsilon_{2} \neq 0, \quad \varepsilon_{1} \varepsilon_{2}=\varepsilon_{2} \varepsilon_{1} \neq 0 .
$$

Hyper-dual numbers form a 4-dimensional vector space over real numbers with basis $\left\{1, \varepsilon_{1}, \varepsilon_{2}, \varepsilon_{1} \varepsilon_{2}\right\}$. Also, a hyper-dual number $D$ can be written as $D=d+d^{*} \varepsilon^{*}$ where $d=a_{0}+a_{1} \varepsilon, d^{*}=a_{2}+a_{3} \varepsilon \in \mathbb{D}$ and $\varepsilon_{1}=\varepsilon, \varepsilon_{2}=\varepsilon^{*}$. The addition and the multiplication of hyper-dual numbers $D_{1}=d_{1}+d_{1}^{*} \varepsilon^{*}$ and $D_{2}=d_{2}+d_{2}^{*} \varepsilon^{*}$ are defined, respectively, as:

$$
D_{1}+D_{2}=\left(d_{1}+d_{2}\right)+\left(d_{1}^{*}+d_{2}^{*}\right) \varepsilon^{*} \quad \text { and } \quad D_{1} D_{2}=d_{1} d_{2}+\left(d_{1} d_{2}^{*}+d_{1}^{*} d_{2}\right) \varepsilon^{*} .
$$

For applications of hyper-dual numbers, see $[4,5,8]$.

Recently, Aslan et al. [1] have defined the hyper-dual split quaternions as

$$
\widehat{q}=D_{0}+D_{1} i+D_{2} j+D_{3} k
$$

where $D_{0}, D_{1}, D_{2}, D_{3} \in \mathbb{H} \mathbb{D}$ and $i, j, k$ satisfy the split quaternion multiplication rules $i^{2}=-1, j^{2}=k^{2}=i j k=1$. It is clear to see that a hyper-dual quaternion is constructed from sixteen real parameters. For the algebraic properties and the geometric interpretations of hyper-dual split quaternions, we refer to [1].

On the other hand, Horadam [11] introduced the quaternions whose components are Fibonacci numbers. More generally, by using Horadam's approach, Halıcı and Karatas [9] defined the $n$th Horadam quaternion as

$$
Q_{w, n}=w_{n}+w_{n+1} i+w_{n+2} j+w_{n+3} k,
$$

where $\left\{w_{n}\right\}$ is the Horadam sequence [12] and is defined by

$$
w_{n}=p w_{n-1}+q w_{n-2}, \quad n \geq 2
$$

with the arbitrary initial values $w_{0}, w_{1}$ and nonzero integers $p, q$. It is clear to see that the Horadam sequence $\left\{w_{n}\right\}:=\left\{w_{n}\left(w_{0}, w_{1} ; p, q\right)\right\}$ generalizes many well known integer sequences such as Fibonacci sequence $\left\{F_{n}\right\}=\left\{w_{n}(0,1 ; 1,1)\right\}$, Lucas sequence $\left\{L_{n}\right\}=\left\{w_{n}(2,1 ; 1,1)\right\}$, generalized Fibonacci sequence $\left\{u_{n}\right\}=\left\{w_{n}(0,1 ; p, q)\right\}$, and the generalized Lucas sequence $\left\{v_{n}\right\}=\left\{w_{n}(2, p ; p, q)\right\}$. The Binet formula of the Horadam sequence $\left\{w_{n}\right\}$ is

$$
w_{n}=\frac{A \alpha^{n}-B \beta^{n}}{\alpha-\beta}
$$

where $A:=w_{1}-w_{0} \beta, B:=w_{1}-w_{0} \alpha$, and $\alpha, \beta$ are the roots of the characteristic polynomial $x^{2}-p x-q$, that is; $\alpha=\frac{\left(p+\sqrt{p^{2}+4 q}\right)}{2}, \beta=\frac{\left(p-\sqrt{p^{2}+4 q}\right)}{2}$. Also we have 
$\alpha \beta=-q, \alpha+\beta=p, \Delta:=\alpha-\beta=\sqrt{p^{2}+4 q}$ with $p^{2}+4 q>0$. The Binet formula for the Horadam quaternions is

$$
Q_{w, n}=\frac{A \alpha^{*} \alpha^{n}-B \beta^{*} \beta^{n}}{\alpha-\beta}
$$

where $\alpha^{*}=1+\alpha i+\alpha^{2} j+\alpha^{3} k$ and $\beta^{*}=1+\beta i+\beta^{2} j+\beta^{3} k$. For details, see [2,9, 17].

Also, Nurkan and Guven [14] introduced the dual Fibonacci quaternions by taking dual Fibonacci numbers instead of real numbers as coefficients. These numbers can also be seen as dual numbers with Fibonacci quaternion coefficients. A generalization of dual Fibonacci quaternions can be found in [18]. In [13], the author introduced the dual Horadam quaternions as

$$
\widetilde{Q}_{w, n}=\widetilde{w}_{n}+\widetilde{w}_{n+1} i+\widetilde{w}_{n+2} j+\widetilde{w}_{n+3} k,
$$

where $\widetilde{w}_{n}=w_{n}+w_{n+1} \varepsilon$ is the $n$th dual Horadam number. Recently, in [15], the authors defined the hyper-dual numbers whose coefficients are from the sequences $\left\{U_{k n}\right\}$ and $\left\{V_{k n}\right\}$ which reduce to the sequences $\left\{w_{n}(0,1 ; p, 1)\right\}$ and $\left\{w_{n}(2, p ; p, 1)\right\}$ for $k=1$, respectively.

In this paper, motivating the definition of hyper-dual split quaternions in [1], we consider the quaternions whose coefficients are taken from hyper-dual Horadam numbers. To do this, first we define the hyper-dual Horadam numbers, then we introduce the quaternions whose coefficients are taken from those numbers. We give the generating function and the Binet formula for hyper-dual Horadam quaternions. Some algebraic properties of these quaternions such as Vajda's identity, Catalan's identity, Cassini's identity, and d'Ocagne's identity are derived with the aid of the Binet formula. Moreover, we develop some matrix identities involving the hyper-dual Horadam quaternions which allow us to obtain some properties of these quaternions.

\section{MAin Results}

In this section, first we define hyper-dual Horadam numbers, then by using these numbers we introduce hyper-dual Horadam quaternions, and investigate the basic properties of these quaternions.

Definition 1. The $n$th hyper-dual Horadam number is defined as

$$
\widehat{w}_{n}=w_{n}+w_{n+1} \varepsilon_{1}+w_{n+2} \varepsilon_{2}+w_{n+3} \varepsilon_{1} \varepsilon_{2},
$$

where $w_{n}$ is the $n$th Horadam number and $\varepsilon_{1}, \varepsilon_{2}$ are dual units satisfying the multiplication rules in (1.2).

Definition 2. The $n$th hyper-dual Horadam quaternion is defined as

$$
\widehat{Q}_{w, n}=\widehat{w}_{n}+\widehat{w}_{n+1} i+\widehat{w}_{n+2} j+\widehat{w}_{n+3} k,
$$

where $\widehat{w}_{n}$ is the $n$th hyper-dual Horadam number and $i, j, k$ satisfy the quaternion multiplication rules in (1.1). 
In the following table, we give the types of quaternions which are mentioned in this paper.

\begin{tabular}{|ll|}
\hline Type of quaternion & Definition \\
\hline \hline Horadam quaternion [9] & $\begin{array}{l}Q_{w, n}=w_{n}+w_{n+1} i+w_{n+2} j+w_{n+3} k, \\
w_{n} \text { is the } n \text {th Horadam number }\end{array}$ \\
Dual Horadam quaternion [13] & $\begin{array}{l}\widetilde{Q}_{w, n}=\widetilde{w}_{n}+\widetilde{w}_{n+1} i+\widetilde{w}_{n+2} j+\widetilde{w}_{n+3} k, \\
\widetilde{w}_{n} \text { is the } n \text {th dual Horadam number }\end{array}$ \\
Hyper-dual Horadam quaternion & $\widehat{Q}_{w, n}=\widehat{w}_{n}+\widehat{w}_{n+1} i+\widehat{w}_{n+2} j+\widehat{w}_{n+3} k$, \\
& \\
& Table 1 \\
\hline
\end{tabular}

Note that the $n$th hyper-dual Horadam quaternion can be expressed as

$$
\widehat{Q}_{w, n}=Q_{w, n}+Q_{w, n+1} \varepsilon_{1}+Q_{w, n+2} \varepsilon_{2}+Q_{w, n+3} \varepsilon_{1} \varepsilon_{2},
$$

where $Q_{w, n}$ is the $n$th Horadam quaternion, and $\varepsilon_{1}, \varepsilon_{2}$ are dual units. The addition and the multiplication of two hyper-dual Horadam quaternions $\widehat{Q}_{w, n}$ and $\widehat{Q}_{w, m}$ are defined as

$$
\begin{aligned}
\widehat{Q}_{w, n}+\widehat{Q}_{w, m}= & \left(Q_{w, n}+Q_{w, m}\right)+\left(Q_{w, n+1}+Q_{w, m+1}\right) \varepsilon_{1} \\
& +\left(Q_{w, n+2}+Q_{w, m+2}\right) \varepsilon_{2}+\left(Q_{w, n+3}+Q_{w, m+3}\right) \varepsilon_{1} \varepsilon_{2} \\
\widehat{Q}_{w, n} \widehat{Q}_{w, m}= & Q_{w, n} Q_{w, m}+\left(Q_{w, n} Q_{w, m+1}+Q_{w, n+1} Q_{w, m}\right) \varepsilon_{1} \\
& +\left(Q_{w, n} Q_{w, m+2}+Q_{w, n+2} Q_{w, m}\right) \varepsilon_{2} \\
& +\left(Q_{w, n} Q_{w, m+3}+Q_{w, n+1} Q_{w, m+2}+Q_{w, n+2} Q_{w, m+1}+Q_{w, n+3} Q_{w, m}\right) \varepsilon_{1} \varepsilon_{2},
\end{aligned}
$$

respectively.

The norm of a hyper-dual Horadam quaternion $\widehat{Q}_{w, n}$ is defined as

$$
N\left(\widehat{Q}_{w, n}\right):=\widehat{Q}_{w, n} \widehat{\widehat{Q}}_{w, n}=\widehat{\widehat{Q}}_{w, n} \widehat{Q}_{w, n}=\widehat{w}_{n}^{2}+\widehat{w}_{n+1}^{2}+\widehat{w}_{n+2}^{2}+\widehat{w}_{n+3}^{2},
$$

where $\widehat{Q}_{w, n}:=\widehat{w}_{n}-\widehat{w}_{n+1} i-\widehat{w}_{n+2} j-\widehat{w}_{n+3} k$ is the conjugate of $\widehat{Q}_{w, n}$. Also the norm of $\widehat{Q}_{w, n}$ can be obtained by the determinant of the matrix

$$
\left(\begin{array}{cc}
\widehat{w}_{n}+\widehat{w}_{n+1} i & -\widehat{w}_{n+2} j-\widehat{w}_{n+3} i \\
\widehat{w}_{n+2} j-\widehat{w}_{n+3} i & \widehat{w}_{n}-\widehat{w}_{n+1} i
\end{array}\right) .
$$

Theorem 1. The hyper-dual Horadam quaternions satisfy the following relation:

$$
\widehat{Q}_{w, n}=p \widehat{Q}_{w, n-1}+q \widehat{Q}_{w, n-2}, \quad n \geq 2 .
$$


Proof. From the definition of the hyper-dual Horadam quaternions and the Horadam quaternions, we have

$$
\begin{aligned}
p \widehat{Q}_{w, n-1}+q \widehat{Q}_{w, n-2}= & p\left(Q_{w, n-1}+Q_{w, n} \varepsilon_{1}+Q_{w, n+1} \varepsilon_{2}+Q_{w, n+2} \varepsilon_{1} \varepsilon_{2}\right) \\
& +q\left(Q_{w, n-2}+Q_{w, n-1} \varepsilon_{1}+Q_{w, n} \varepsilon_{2}+Q_{w, n+1} \varepsilon_{1} \varepsilon_{2}\right) \\
= & \left(p Q_{w, n-1}+q Q_{w, n-2}\right)+\left(p Q_{w, n}+q Q_{w, n-1}\right) \varepsilon_{1} \\
& +\left(p Q_{w, n+1}+q Q_{w, n}\right) \varepsilon_{2}+\left(p Q_{w, n+2}+q Q_{w, n+1}\right) \varepsilon_{1} \varepsilon_{2} \\
= & Q_{w, n}+Q_{w, n+1} \varepsilon_{1}+Q_{w, n+2} \varepsilon_{2}+Q_{w, n+3} \varepsilon_{1} \varepsilon_{2} .
\end{aligned}
$$

Theorem 2. The generating function for hyper-dual Horadam quaternions is

$$
G(x)=\frac{\widehat{Q}_{w, 0}+\left(\widehat{Q}_{w, 1}-p \widehat{Q}_{w, 0}\right) x}{1-p x-q x^{2}} .
$$

Proof. Let

$$
G(x):=\sum_{n=0}^{\infty} \widehat{Q}_{w, n} x^{n}=\widehat{Q}_{w, 0}+\widehat{Q}_{w, 1} x+\sum_{n=2}^{\infty} \widehat{Q}_{w, n} x^{n} .
$$

From Theorem 1, we have

$$
\begin{aligned}
\left(1-p x-q x^{2}\right) G(x)= & \widehat{Q}_{w, 0}+\widehat{Q}_{w, 1} x+\sum_{n=2}^{\infty} \widehat{Q}_{w, n} x^{n} \\
& -p \widehat{Q}_{w, 0} x-p \sum_{n=2}^{\infty} \widehat{Q}_{w, n-1} x^{n}-q \sum_{n=2}^{\infty} \widehat{Q}_{w, n-2} x^{n} \\
= & \widehat{Q}_{w, 0}+\widehat{Q}_{w, 1} x-p \widehat{Q}_{w, 0} x+\sum_{n=2}^{\infty}\left(\widehat{Q}_{w, n}-p \widehat{Q}_{w, n-1}-q \widehat{Q}_{w, n-2}\right) x^{n} \\
= & \widehat{Q}_{w, 0}+\left(\widehat{Q}_{w, 1}-p \widehat{Q}_{w, 0}\right) x
\end{aligned}
$$

Thus, we get the desired result.

Theorem 3. The Binet formula of hyper-dual Horadam quaternions is

$$
\widehat{Q}_{w, n}=\frac{A \alpha^{*} \underline{\alpha} \alpha^{n}-B \beta^{*} \underline{\beta} \underline{\beta}^{n}}{\alpha-\beta},
$$

where

$$
\begin{aligned}
\alpha^{*} & =1+\alpha i+\alpha^{2} j+\alpha^{3} k, & \beta^{*} & =1+\beta i+\beta^{2} j+\beta^{3} k, \\
\underline{\alpha} & =1+\alpha \varepsilon_{1}+\alpha^{2} \varepsilon_{2}+\alpha^{3} \varepsilon_{1} \varepsilon_{2}, & \underline{\beta} & =1+\beta \varepsilon_{1}+\beta^{2} \varepsilon_{2}+\beta^{3} \varepsilon_{1} \varepsilon_{2} .
\end{aligned}
$$


Proof. From the Binet formula of Horadam quaternions in (1.3), we have

$$
\begin{aligned}
\widehat{Q}_{w, n}= & Q_{w, n}+Q_{w, n+1} \varepsilon_{1}+Q_{w, n+2} \varepsilon_{2}+Q_{w, n+3} \varepsilon_{1} \varepsilon_{2} \\
= & \left(\frac{A \alpha^{*} \alpha^{n}-B \beta^{*} \beta^{n}}{\alpha-\beta}\right)+\left(\frac{A \alpha^{*} \alpha^{n+1}-B \beta^{*} \beta^{n+1}}{\alpha-\beta}\right) \varepsilon_{1} \\
& +\left(\frac{A \alpha^{*} \alpha^{n+2}-B \beta^{*} \beta^{n+2}}{\alpha-\beta}\right) \varepsilon_{2}+\left(\frac{A \alpha^{*} \alpha^{n+3}-B \beta^{*} \beta^{n+3}}{\alpha-\beta}\right) \varepsilon_{1} \varepsilon_{2} \\
= & \frac{A \alpha^{*} \alpha^{n}}{\alpha-\beta}\left(1+\alpha \varepsilon_{1}+\alpha^{2} \varepsilon_{2}+\alpha^{3} \varepsilon_{1} \varepsilon_{2}\right)-\frac{B \beta^{*} \beta^{n}}{\alpha-\beta}\left(1+\beta \varepsilon_{1}+\beta^{2} \varepsilon_{2}+\beta^{3} \varepsilon_{1} \varepsilon_{2}\right) \\
= & \frac{A \alpha^{*} \underline{\alpha} \alpha^{n}-B \beta^{*} \underline{\beta} \beta^{n}}{\alpha-\beta} .
\end{aligned}
$$

From Theorem 3, the Binet formulas of the hyper-dual generalized Fibonacci and Lucas quaternions can be obtained as

$$
\widehat{Q}_{u, n}=\frac{\alpha^{*} \underline{\alpha} \alpha^{n}-\beta^{*} \underline{\beta} \beta^{n}}{\alpha-\beta} \quad \text { and } \quad \widehat{Q}_{v, n}=\alpha^{*} \underline{\alpha} \alpha^{n}+\beta^{*} \underline{\beta} \beta^{n},
$$

respectively.

By using (2), we obtain the following relation between the hyper-dual Fibonacci quaternions and the hyper-dual Lucas quaternions.

Theorem 4. Let $n$ be a positive integer. For hyper-dual Fibonacci quaternions and hyper-dual Lucas quaternions, the following equality holds:

$$
\widehat{Q}_{v, n}=\widehat{Q}_{u, n+1}+q \widehat{Q}_{u, n-1} .
$$

Now we need the following lemma which allows us a remarkable simplification for obtaining the properties of hyper-dual Horadam quaternions.

Lemma 1. Let $\theta:=1-q+q^{2}-q^{3}$ and $\omega:=(1-q) i+\left(1+p^{2}+q\right) k$. Then we have

$$
\begin{aligned}
\underline{\alpha \beta} & =\widehat{v}_{0}-\left(1+p q \varepsilon_{1} \varepsilon_{2}\right), \\
\alpha^{*} \beta^{*} & =Q_{v, 0}-\theta-\Delta q\left(Q_{u, 0}-\omega\right), \\
\beta^{*} \alpha^{*} & =Q_{v, 0}-\theta+\Delta q\left(Q_{u, 0}-\omega\right) .
\end{aligned}
$$

Proof. The proof can be done by using the multiplication rules in (1.1) and (1.2). We should note that the set of hyper-dual numbers form a commutative algebra. Therefore we have $\underline{\alpha} \beta=\beta \underline{\alpha}$. But since the quaternion multiplication is noncommutative, $\alpha^{*} \beta^{*}$ need not be equal to $\beta^{*} \alpha^{*}$. The results for $\alpha^{*} \beta^{*}$ and $\beta^{*} \alpha^{*}$ can also be found in [2, Lemma 1]. 
Theorem 5. (Vajda's Identity) For integers $n, r$, and $s$, we have

$$
\begin{aligned}
\widehat{Q}_{w, n+r} \widehat{Q}_{w, n+s}-\widehat{Q}_{w, n} \widehat{Q}_{w, n+r+s}= & A B(-q)^{n}\left(\widehat{v}_{0}-\left(1+p q \varepsilon_{1} \varepsilon_{2}\right)\right) \\
& \cdot u_{r}\left(\left(Q_{v, 0}-\theta\right) u_{s}+q\left(Q_{u, 0}-\omega\right) v_{s}\right) .
\end{aligned}
$$

Proof. From the Binet formula of hyper-dual Horadam quaternions, we have

$$
\begin{aligned}
\Delta^{2} & \left(\widehat{Q}_{w, n+r} \widehat{Q}_{w, n+s}-\widehat{Q}_{w, n} \widehat{Q}_{w, n+r+s}\right)= \\
= & \left(A \alpha^{*} \underline{\alpha} \alpha^{n+r}-B \beta^{*} \underline{\beta} \beta^{n+r}\right)\left(A \alpha^{*} \underline{\alpha} \alpha^{n+s}-B \beta^{*} \underline{\beta} \beta^{n+s}\right) \\
& -\left(A \alpha^{*} \underline{\alpha} \alpha^{n}-B \beta^{*} \underline{\beta} \underline{\beta}^{n}\right)\left(A \alpha^{*} \underline{\alpha} \alpha^{n+r+s}-B \beta^{*} \underline{\beta} \beta^{n+r+s}\right) \\
= & A^{2}\left(\alpha^{*} \underline{\alpha}\right)^{2} \alpha^{2 n+r+s}-A B \alpha^{*} \beta^{*} \underline{\alpha} \underline{\beta} \alpha^{n+r} \beta^{n+s}-A B \beta^{*} \alpha^{*} \underline{\beta} \underline{\alpha} \alpha^{n+s} \beta^{n+r} \\
& +B^{2}\left(\beta^{*} \underline{\beta}\right)^{2} \beta^{2 n+r+s}-A^{2}\left(\alpha^{*} \underline{\alpha}\right)^{2} \alpha^{2 n+r+s}+A B \alpha^{*} \beta^{*} \underline{\alpha} \underline{\beta} \alpha^{n} \beta^{n+r+s} \\
& +A B \beta^{*} \alpha^{*} \underline{\beta} \underline{\alpha} \beta^{n} \alpha^{n+r+s}-B^{2}\left(\beta^{*} \underline{\beta}\right)^{2} \beta^{2 n+r+s} \\
= & A B(\alpha \beta)^{n} \underline{\alpha} \underline{\beta}\left(\alpha^{*} \beta^{*}\left(-\alpha^{r} \beta^{s}+\beta^{r+s}\right)+\beta^{*} \alpha^{*}\left(-\alpha^{s} \beta^{r}+\alpha^{r+s}\right)\right) .
\end{aligned}
$$

By using Lemma 1 , we have

$$
\begin{aligned}
& \widehat{Q}_{w, n+r} \widehat{Q}_{w, n+s}-\widehat{Q}_{w, n} \widehat{Q}_{w, n+r+s}= \\
&=\frac{A B}{\Delta^{2}}(-q)^{n} \underline{\alpha} \underline{\beta}\left(-\alpha^{*} \beta^{*} \beta^{s}\left(\alpha^{r}-\beta^{r}\right)+\beta^{*} \alpha^{*} \alpha^{s}\left(\alpha^{r}-\beta^{r}\right)\right) \\
&=\frac{A B}{\Delta}(-q)^{n} \underline{\alpha} \underline{\beta} u_{r}\left(\beta^{*} \alpha^{*} \alpha^{s}-\alpha^{*} \beta^{*} \beta^{s}\right) \\
&=\frac{A B}{\Delta}(-q)^{n} \underline{\alpha} \underline{\beta} u_{r}\left(Q_{v, 0}-\theta+\Delta q\left(Q_{u, 0}-\omega\right)\right) \alpha^{s} \\
&-\frac{A B}{\Delta}(-q)^{n} \underline{\alpha} \underline{\beta} u_{r}\left(Q_{v, 0}-\theta-\Delta q\left(Q_{u, 0}-\omega\right)\right) \beta^{s} \\
&= A B(-q)^{n}\left(\widehat{v}_{0}-\left(1+p q \varepsilon_{1} \varepsilon_{2}\right)\right) u_{r}\left(\left(Q_{v, 0}-\theta\right) u_{s}+q\left(Q_{u, 0}-\omega\right) v_{s}\right) .
\end{aligned}
$$

From Theorem 5, we have the following results:

If we set $r \rightarrow-s$, we get the following Catalan's identity for hyper-dual Horadam quaternions:

$$
\begin{gathered}
\widehat{Q}_{w, n-s} \widehat{Q}_{w, n+s}-\widehat{Q}_{w, n}^{2}=-A B(-q)^{n-s}\left(\widehat{v}_{0}-\left(1+p q \varepsilon_{1} \varepsilon_{2}\right)\right) u_{s}\left(\left(Q_{v, 0}-\theta\right) u_{s}\right. \\
\left.+q\left(Q_{u, 0}-\omega\right) v_{s}\right) .
\end{gathered}
$$

Here note that $u_{-n}=\frac{-u_{n}}{(-q)^{n}}$.

If we set $s=-r=1$, we get the following Cassini's identity for hyper-dual Horadam quaternions: 


$$
\begin{aligned}
& \widehat{Q}_{w, n-1} \widehat{Q}_{w, n+1}-\widehat{Q}_{w, n}^{2}= \\
& \quad=-A B(-q)^{n-1}\left(\widehat{v}_{0}-\left(1+p q \varepsilon_{1} \varepsilon_{2}\right)\right)\left(Q_{v, 0}-\theta+p q\left(Q_{u, 0}-\omega\right)\right) .
\end{aligned}
$$

If we set $s \rightarrow m-n$, and fix $r=1$, we get the following d'Ocagne's identity for hyper-dual Horadam quaternions:

$$
\begin{aligned}
& \widehat{Q}_{w, n+1} \widehat{Q}_{w, m}-\widehat{Q}_{w, n} \widehat{Q}_{w, m+1}= \\
& \quad=A B(-q)^{n}\left(\widehat{v}_{0}-\left(1+p q \varepsilon_{1} \varepsilon_{2}\right)\right)\left(\left(Q_{v, 0}-\theta\right) u_{m-n}+q\left(Q_{u, 0}-\omega\right) v_{m-n}\right) .
\end{aligned}
$$

Next, we give some summation formulas for hyper-dual Horadam quaternions.

Theorem 6. For $n \geq 2$, we have

$$
\sum_{r=1}^{n-1} \widehat{Q}_{w, r}=\frac{\widehat{Q}_{w, n}-\widehat{Q}_{w, 1}+q\left(\widehat{Q}_{w, n-1}-\widehat{Q}_{w, 0}\right)}{p+q-1} .
$$

Proof. From the Binet formula for hyper-dual Horadam quaternions, we have

$$
\begin{aligned}
\sum_{r=1}^{n-1} \widehat{Q}_{w, r}= & \sum_{r=1}^{n-1} \frac{A \alpha^{*} \underline{\alpha} \alpha^{r}-B \beta^{*} \underline{\beta} \underline{\beta}^{r}}{\alpha-\beta}=\frac{A \alpha^{*} \underline{\alpha}}{\alpha-\beta} \sum_{r=1}^{n-1} \alpha^{r}-\frac{B \beta^{*} \underline{\beta}}{\alpha-\beta} \sum_{r=1}^{n-1} \beta^{r} \\
= & \frac{A \alpha^{*} \underline{\alpha}}{\alpha-\beta}\left(\frac{\alpha^{n}-\alpha}{\alpha-1}\right)-\frac{B \beta^{*} \underline{\beta}}{\alpha-\beta}\left(\frac{\beta^{n}-\beta}{\beta-1}\right) \\
= & \frac{1}{(\alpha-\beta)(1-p-q)}\left(-\left(A \alpha^{*} \underline{\alpha} \alpha^{n}-B \beta^{*} \underline{\beta} \beta^{n}\right)\right. \\
& -q\left(A \alpha^{*} \underline{\alpha} \alpha^{n-1}-B \beta^{*} \underline{\beta} \beta^{n-1}\right)+q\left(A \alpha^{*} \underline{\alpha}-B \beta^{*} \underline{\beta}\right) \\
& \left.+\left(A \alpha^{*} \underline{\alpha} \alpha-B \beta^{*} \underline{\beta} \beta\right)\right) \\
= & \frac{-\widehat{Q}_{w, n}-q \widehat{Q}_{w, n-1}+q \widehat{Q}_{w, 0}+\widehat{Q}_{w, 1}}{1-p-q} .
\end{aligned}
$$

Theorem 7. For nonnegative integers $n$ and $r$, we have

$$
\sum_{m=0}^{n}\left(\begin{array}{c}
n \\
m
\end{array}\right) q^{n-m} p^{m} \widehat{Q}_{w, m+r}=\widehat{Q}_{w, 2 n+r} .
$$

Proof. From the Binet formula for hyper-dual Horadam quaternions, we have

$$
\begin{aligned}
& \sum_{m=0}^{n}\left(\begin{array}{c}
n \\
m
\end{array}\right) q^{n-m} p^{m} \widehat{Q}_{w, m+r}=\sum_{m=0}^{n}\left(\begin{array}{c}
n \\
m
\end{array}\right) q^{n-m} p^{m}\left(\frac{A \alpha^{*} \underline{\alpha} \alpha^{m+r}-B \beta^{*} \underline{\beta} \underline{\beta}^{m+r}}{\alpha-\beta}\right)= \\
& =\frac{A \alpha^{*} \underline{\alpha} \alpha^{r}}{\alpha-\beta} \sum_{m=0}^{n}\left(\begin{array}{c}
n \\
m
\end{array}\right) q^{n-m}(p \alpha)^{m}-\frac{B \beta^{*} \underline{\beta} \beta^{r}}{\alpha-\beta} \sum_{m=0}^{n}\left(\begin{array}{c}
n \\
m
\end{array}\right) q^{n-m}(p \beta)^{m}
\end{aligned}
$$




$$
\begin{aligned}
& =\frac{A \alpha^{*} \underline{\alpha} \alpha^{r}}{\alpha-\beta}(q+p \alpha)^{n}-\frac{B \beta^{*} \underline{\beta} \beta^{r}}{\alpha-\beta}(q+p \beta)^{n} \\
& =\frac{A \alpha^{*} \underline{\alpha} \alpha^{2 n+r}-B \beta^{*} \underline{\beta} \beta^{2 n+r}}{\alpha-\beta}=\widehat{Q}_{w, 2 n+r} .
\end{aligned}
$$

Finally, we give some matrix representations for hyper-dual Horadam quaternions, and derive some properties of hyper-dual Horadam quaternions by using matrix approach.

Let define the matrices $\mathbf{U}:=\left[\begin{array}{cc}p & q \\ 1 & 0\end{array}\right], \mathbf{W}_{n}:=\left[\begin{array}{cc}w_{n+2} & q w_{n+1} \\ w_{n+1} & q w_{n}\end{array}\right]$. It is well-known that for the Horadam numbers, we have the matrix equality:

$$
\mathbf{W}_{0} \mathbf{U}^{n}=\mathbf{W}_{n} .
$$

For details, see [17]. Now let define the matrix $\mathbf{M}_{w, n}:=\left[\begin{array}{cc}\widehat{Q}_{w, n+2} & q \widehat{Q}_{w, n+1} \\ \widehat{Q}_{w, n+1} & q \widehat{Q}_{w, n}\end{array}\right]$. Considering the relation (2.2), we have the matrix equalities

$$
\mathbf{M}_{w, 0} \mathbf{U}^{n}=\mathbf{M}_{w, n}
$$

and

$$
\mathbf{M}_{u, 0}\left(\mathbf{W}_{0} \mathbf{U}^{n-1}\right)=\mathbf{M}_{w, n},
$$

which can be proven by using induction.

Theorem 8. For integers $n, m \geq 1$, we have the following equalities:

$$
\begin{aligned}
& \widehat{Q}_{w, m+n}=\widehat{Q}_{w, m+1} u_{n}+q \widehat{Q}_{w, m} u_{n-1}, \\
& \widehat{Q}_{w, m+n}=w_{n} \widehat{Q}_{u, m+1}+q w_{n-1} \widehat{Q}_{u, m} .
\end{aligned}
$$

Proof. From the matrix equality $\mathbf{M}_{w, 0} \mathbf{U}^{m+n-2}=\left(\mathbf{M}_{w, 0} \mathbf{U}^{m-1}\right) \mathbf{U}^{n-1}$, we have

$$
\left[\begin{array}{cc}
\widehat{Q}_{w, m+n} & q \widehat{Q}_{w, m+n-1} \\
\widehat{Q}_{w, m+n-1} & q \widehat{Q}_{w, m+n-2}
\end{array}\right]=\left[\begin{array}{cc}
\widehat{Q}_{w, m+1} & q \widehat{Q}_{w, m} \\
\widehat{Q}_{w, m} & q \widehat{Q}_{w, m-1}
\end{array}\right]\left[\begin{array}{cc}
u_{n} & q u_{n-1} \\
u_{n-1} & q u_{n-2}
\end{array}\right] .
$$

By equating the corresponding entries of both sides of the matrix equation, we get the identity (2.5).

Now consider the matrix equality $\mathbf{M}_{u, 0}\left(\mathbf{W}_{0} \mathbf{U}^{m+n-2}\right)=\left(\mathbf{W}_{0} \mathbf{U}^{n-1}\right) \mathbf{U}^{m-1} \mathbf{M}_{u, 0}$. Then we have

$$
\left[\begin{array}{cc}
\widehat{Q}_{w, m+n+1} & q \widehat{Q}_{w, m+n} \\
\widehat{Q}_{w, m+n} & q \widehat{Q}_{w, m+n-1}
\end{array}\right]=\left[\begin{array}{cc}
w_{n+1} & q w_{n} \\
w_{n} & q w_{n-1}
\end{array}\right]\left[\begin{array}{cc}
\widehat{Q}_{u, m+1} & q \widehat{Q}_{u, m} \\
\widehat{Q}_{u, m} & q \widehat{Q}_{u, m-1}
\end{array}\right]
$$

Similarly, by equating the corresponding entries of both sides of the above matrix equation, we get the desired result in (2.6). 
From the matrix equalities (2.3) and (2.4), one can obtain several results for the hyper-dual Horadam quaternions. For example, if we take the determinant of the both side of this matrix identity (2.3), then we get the Cassini's identity in terms of hyper-dual Horadam quaternions as:

$$
\widehat{Q}_{w, n-1} \widehat{Q}_{w, n+1}-\widehat{Q}_{w, n}^{2}=(-q)^{n-1}\left(\widehat{Q}_{w, 0} \widehat{Q}_{w, 2}-\widehat{Q}_{w, 1}^{2}\right) .
$$

Note that different from the identity (2.1), here the right hand side of the equation (2.7) is expressed in terms of only the hyper-dual Horadam quaternions.

\section{CONCLUSION}

In this paper, we define quaternions whose components are hyper-dual Horadam numbers. The main advantage of introducing hyper-dual Horadam quaternions is that many hyper-dual numbers with celebrated numbers such as Fibonacci, Lucas, Pell, Pell-Lucas, Jacosthal, Jacobsthal-Lucas numbers can be deduced as particular cases of hyper-dual Horadam quaternions. We give the generating function and the Binet formula for these quaternions. With the help of the Binet formula of hyperdual Horadam quaternions, we derive many properties of these quaternions such as summation formulas, binomial sum identities, Vajda's identity, Catalan's identity, Cassini identity, and d'Ocagne's identity. Also by means of the matrix representation of hyper-dual Horadam quaternions, we examine several identities for these quaternions. The algebra of quaternions is noncommutative, whereas the algebra of hyper-dual numbers is commutative. So, it is interesting to study special type of numbers which are involving both quaternionic and hyper-dual units. For the interested readers, the results of this paper could be applied for higher order hyper-dual numbers which were given in [8].

\section{ACKNOWLEDGEMENT}

The authors would like to thank the anonymous reviewer for his/her valuable comments and suggestions.

\section{REFERENCES}

[1] S. Aslan, M. Bekar, and Y. Yayll, "Hyper-dual split quaternions and rigid body motion," J. Geom. Phys., vol. 158, p. 12, 2020, id/No 103876, doi: 10.1016/j.geomphys.2020.103876.

[2] G. Cerda-Morales, "Some properties of (p,q)-Fibonaccin and Horadam quaternions," Commun.Fac.Sci.Univ.Ank.Ser.Al Math. Stat., vol. 69, no. 2, pp. 1104-1110, 2020, doi: 10.31801/cfsuasmas.696617.

[3] W. Clifford, "Preliminary sketch of biquaternions," Proceedings of London Mathematical Society, vol. 4, pp. 361-395, 1873, doi: 10.1112/plms/s1-4.1.381.

[4] A. Cohen and M. Shoham, "Application of hyper-dual numbers to multi-body kinematics." $J$. Mech. Rob., vol. 8, no. 1, p. 011005, 2015, doi: 10.1115/1.4030588. 
[5] A. Cohen and M. Shoham, "Application of hyper-dual numbers to rigid bodies equations of motion." Mech. Mach. Theory, vol. 111, pp. 76-84, 2017, doi: 10.1016/j.mechmachtheory.2017.01.013.

[6] J. Fike, "Numerically exact derivative calculations using hyper-dual numbers," 3rd Annual Student Joint Workshop in Simulation-Based 26 Engineering and Design, 2009.

[7] J. Fike and J. Alonso, "The development of hyper-dual numbers for exact second-derivative calculations," 49th AIAA Aerodpace Sciences Meeting 28 including the New Horizons Forum and Aerospace Exposition, Orlando, Florida, pp. 4-7, 2011, doi: 10.2514/6.2011-886.

[8] J. Fike and J.J.Alonso, "Principle of transference - An extension to hyper-dual numbers," Mechanism and Machine Theory, vol. 125, pp. 101-110, 2018, doi: 10.1016/j.mechmachtheory.2017.12.007.

[9] S. Halici and A. Karataş, "On a generalization for Fibonacci quaternions," Chaos Solitons Fractals, vol. 98, pp. 178-182, 2017, doi: 10.1016/j.chaos.2017.03.037.

[10] W. Hamilton, Lectures on quaternions. Dublin: Hodges and Smith, 1853.

[11] A. F. Horadam, "Complex Fibonacci numbers and Fibonacci quaternions," Am. Math. Mon., vol. 70, pp. 289-291, 1963, doi: 10.2307/2313129.

[12] A. F. Horadam, "Basic properties of a certain generalized sequence of numbers," Fibonacci Q., vol. 3, pp. 161-176, 1965.

[13] N. Kilic, "On dual Horadam quaternions," An. Ştiinţ. Univ. Al. I. Cuza Iaşi, Ser. Nouă, Mat., vol. 66, no. 1, pp. 101-112, 2020.

[14] S. K. Nurkan and I. A. Güven, "Dual Fibonacci quaternions," Adv. Appl. Clifford Algebr., vol. 25, no. 2, pp. 403-414, 2015, doi: 10.1007/s00006-014-0488-7.

[15] N. Omur and S. Koparal, "On hyper-dual generalized Fibonacci numbers," Notes on Number Theory and Discrete Mathematics, vol. 26, no. 1, pp. 191-198, 2020, doi: 10.7546/nntdm.2020.26.1.191-198.

[16] E. Study, Geometry der Dynamen. Leipzig: Wiley, 1901.

[17] E. Tan and H. Leung, "Some results on Horadam quaternions," Chaos, Solitons and Fractals, vol. 138, no. 109961, 2020, doi: doi.org/10.1016/j.chaos.2020.109961.

[18] E. Tan and I. Gök, "A generalization of dual bi-periodic Fibonacci quaternions," J. Math. Ext., vol. 13, no. 1, pp. 67-81, 2019.

Authors' addresses

N. Rosa Ait-Amrane

USTHB, Faculty of Mathematics, RECITS Laboratory, 16111 Bab-Ezzouar, Algiers, Algeria

E-mail address: rosamrane@gmail.com

\section{Ismail Gok}

Department of Mathematics, Ankara University, Science Faculty, 06100 Tandogan Ankara, Turkey

E-mail address: igokescience.ankara.edu.tr

Elif Tan

(Corresponding author) Department of Mathematics, Ankara University, Science Faculty, 06100 Tandogan Ankara, Turkey.

E-mail address: etan@ankara.edu.tr 